\title{
Trait-Based Models of Decomposition with Interspecific and Environmental Interactions
}

\author{
SiYu Liu ${ }^{1, a^{*}}$, RuiXi Wang ${ }^{2, b^{*}}$, and LuYao Dong ${ }^{3, \mathrm{c}^{*}}$ \\ ${ }^{1}$ School of Mechatronic Engineering, Beijing Institute of Technology, Beijing, China \\ ${ }^{2}$ School of Mechatronic Engineering, Beijing Institute of Technology, Beijing, China \\ ${ }^{3}$ School of Aerospace Engineering, Beijing Institute of Technology, Beijing, China
}

\begin{abstract}
Most saprotrophic fungi are dominant decomposers, and they play a vital role in the carbon cycle and stability of ecosystem. Previous researchers examined that how traits and environments influence the decomposition rate(DR), but few studies last long enough to scale short-term results up to long-term trends. In this paper, we build several trait-based models of decomposition with interspecific and environmental interactions to describe both short- and long-term trends. First, we build the model of decomposition ratio versus time with covariates - hyphal extension ratio and moisture tolerance for each species. Second, we define Interspecific Interference Ratio (IIR) to reflect interactions between fungi species and rank the relative competitiveness based on Grey System Theory with four evaluation indices: hyphal extension rate, moisture niche width, the hyphal density and thermal niche width. Then, we predict the relative advantages and disadvantages for different species by IIR and analyze the impact of biodiversity. Finally, we perform sensitivity and robustness analysis of our models, which exhibits the wide prospects and a deeper understanding of the role of fungi in the ecosystem.
\end{abstract}

\section{Introduction}

Fungi vary greatly in their decomposing ability. Some researchers used trait-based approach to quantify the niches of 23 species of saprotrophic wood decomposer fungi and explored the relationship between different traits and the decomposing rate of wood ${ }^{[1]}$ (D. S. Maynard et al, 2019). The strongest single predictor of wood decomposition is the hyphal extension rate (Nicky Lustenhouwer, Daniel S. Maynard et al, 2020). The hyphal are branching filaments which are able to enter organic matter, and it was found that if the hyphal extension rate was larger, the fungus decomposes faster. Another important trait is called moisture tolerance. Researchers found that if fungi were better able to adapt to a large range of moisture, they tended to decompose wood slower ${ }^{[1]}$ (Nicky Lustenhouwer, Daniel S. Maynard et al, 2020). Besides, environmental conditions such as arid, temperate, tropic rain forest also have impact on the decomposition of fungi. Biodiversity of fungal communities of a system can influence the overall decomposing efficiency as well ${ }^{[2]}$ (Krause, Sascha et al.2014).

\section{Decomposition rate of multiple fungi species}

\subsection{Model description}

In this model, we focus on the saprotrophic wood decomposer fungi including 20 unique species (34 isolates) and measure both physiological growth traits and ecological performance traits. From the previous experiments, we knew that hyphal extension rate of the fungal colony is the strongest predictor of wood decomposition, so we choose hyphal extension rate, also called growth rates, to represent physiological growth traits of fungi. According to the laboratory assays, several biochemical and ecological traits correlated negatively with the decomposition rate, for example, fungi that were tolerant of a wider range of moisture had lower decomposition rate ${ }^{[1]}$, so moisture tolerance is used as one ecological performance trait in our model.

\subsection{Mathematic model}

Using Weibull function to represent decay in the experiments, we define the proportion of the remaining mass $\left(M_{\text {remain }}\right)$ per unit as follows:

\footnotetext{
* These authors contributed equally to this work

*Corresponding author: ${ }^{\text {a } 1098782848 @ q q . c o m, ~}{ }^{\text {bLexi.RX.Wang@outlook.com, }{ }^{\mathrm{c} d l y} 114 \mathrm{~s} @ 163 . c o m}$
} 


$$
M_{\text {remain }}(t)=1-e^{-(\lambda t)^{c}}
$$

where $\lambda$ is the scale factor and $\mathrm{c}$ is the shape factor.

When $c>1$, mass loss accelerates. When $c<1$, mass loss will decelerate through time. We choose $c=1$ in this model, so the remaining mass satisfies NegExp model, and the decomposing mass can be expressed as:

$$
M_{d e}(t)=1-M_{\text {remain }}(t)=e^{-\lambda t}
$$

Decomposition rate can be expressed as:

$$
R_{d e}=\frac{d M_{d e}}{d t}=-\lambda e^{-\lambda t}
$$

To estimate the scale factor $\lambda$, we employed a double log link function and normally distributed measurementlevel error on the transformed scale ${ }^{[2]}$ (Oberle et al, 2016). $\lambda$ may vary with different traits and is treated as a stochastic variable.

We consider that $\lambda$ obeys logarithmic normal distribution, so we can use expectation to estimate the average value of $\lambda$. As mentioned before, decomposition rate is influenced by two traits of fungi in this model, so we use multivariable regression and gradient descent algorithm to express the linear combination of hyphal extension rate and moisture tolerance and yield the $\lambda$ :

$$
\ln \lambda=\omega_{1} \cdot R_{\text {grow }}+\omega_{2} \cdot \text { Tol }_{\text {moist }}+c_{0}
$$

We can also express in matrix:

$$
\ln \lambda=\omega^{T} x_{i}+c_{0}
$$

where $\omega=\left[\begin{array}{c}\omega_{1} \\ \omega_{2}\end{array}\right], \quad x_{i}=\left[\begin{array}{c}R_{\text {grow }} \\ \text { Tol }\end{array}\right]$. Then, we can get cost function:

$$
J(\omega, b)=\sum_{i=1}^{2}\left(y_{i}-\omega^{T} x_{i}-c_{0}\right)^{2}
$$

where $y_{i}$ is the real value of $\ln \lambda$.

According to the experimental data(D. S. Maynard et al, 2019), use statistical software to calculate the $S S E$ (sum of squares due to error), after iteration we get that $S S E=$ 0.396 , and SD (standard error) $=0.026$, so the result is reliable. Then, we use least square method to yield:

$$
\lambda=100 e^{0.246 \cdot R_{\text {grow }}-0.683 \cdot \text { Tol }_{\text {moist }}-12.203}
$$

We consider $\lambda$ as a parameter to reflect the effect of multiple species fungi.

Since hyphal extension rate of a fungus $\left(R_{\text {grow }}\right)$ and moisture tolerance ( Tol $_{\text {moist }}$ ) are given in the dataset, we can calculate $\lambda$ and substitute the value into equation (3), then we get the decomposition rate :

$$
R_{d e}=10^{4} \cdot \lambda e^{-\lambda t}
$$

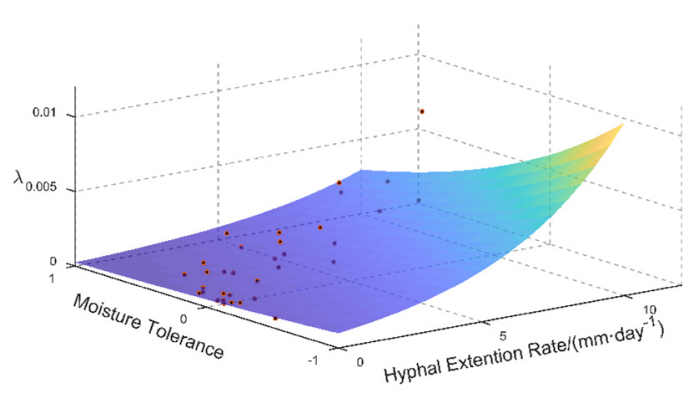

(A)

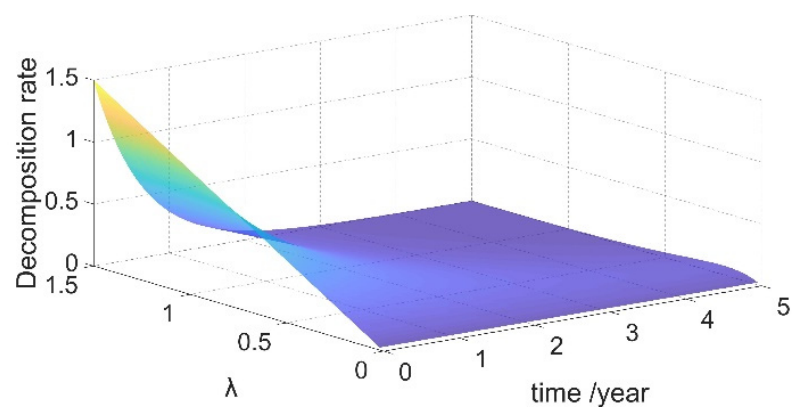

(B)

Fig. 1. (A) The relationship between hyphal extension rate, moisture tolerance and $\lambda$. (B) The relationship between fungi effect $\lambda$ (defined in equation (7)), time and decomposition rate.

In Figure 1(A), we view $\lambda$ as a factor to quantify the effect of fungi in decomposition. Moisture tolerance is the difference of competitive ranking and its moisture niche width. Hyphal extension rate is just the growth rate of fungi. $\lambda$ is linear correlated to these two traits. Discrete points are experimental data based on 20 fungi species (34 isolates included). In Figure 1(B), We use mass loss of materials to measure the decomposition process and assume mass loss is calculated through the integral of deposition rate with respect of time.

\section{Incorporate Interactions Between Fungi Species}

\subsection{Interactions between fungi species}

In the process of decomposition, there exists interactions between fungi with different growth rates and moisture tolerance. According to Principle of competitive exclusion, in competition between species that seek same ecological niche, the more similar the two species are, the more overlapping niches will be, and the more intense competitions will occur. Wood decay fungi in our dataset are quite similar species, and we will reveal the relationship between different species in the following essay.

We use Grey System Theory to rank the competitive power of fungi species. For the system with incomplete information, this theory can be used to study and predict unknown field through known information.

First, we determine the evaluation object and evaluation standard. In our dataset, there are 34 isolates of fungi, and we choose hyphal extension rate, moisture niche width, the hyphal density and thermal niche width as four evaluation indices. Rewrite these species and traits into a sequence:

$$
a_{i}=\left\{a_{i}(k) \mid k=1,2, \ldots, 4\right\}, \quad i=1,2, \ldots, 34
$$

$a_{i}(k)$ is the value of the $i$ th object about the $k^{t h}$ index. As for the evaluation standard, we virtualize index values of the best object, and expressed them in reference sequence:

$$
a_{0}=\left\{a_{0}(k) \mid k=1,2, \ldots, 4\right\}
$$

Second, we calculate grey relational coefficient: 


$$
\begin{gathered}
\xi_{i}(k)=\frac{\min \min \left|a_{0}(t)-a_{s}(t)\right|+\rho \max \max \left|a_{0}(t)-a_{s}(t)\right|}{\left|a_{0}(k)-a_{i}(k)\right|+\rho \max \max \left|a_{0}(t)-a_{s}(t)\right|} \\
i=1,2, \ldots, 34, k=1,2, \ldots, 4
\end{gathered}
$$

where, $\rho \in[0,1], \quad \max \max \left|a_{0}(t)-a_{s}(t)\right|$, $\min \min \left|a_{0}(t)-a_{s}(t)\right|$ is the maximum and minimal difference of two stages respectively. This coefficient represents the correlation between $a_{i}$ and reference sequence on the $k^{t h}$ index.

Then, we yield the Grey Relational Degree (GRD):

$$
r_{i}=\sum_{k=1}^{4} \omega_{k} \xi_{i}(k), \quad i=1,2, \ldots, 34
$$

where $\omega_{k}$ is the weight of the evaluation indices, and we consider that four traits are equally weighted.

Suppose that under given environment, $n$ species have a chance of survival, which are expressed as a set $B=\left\{b_{i}\right\}$ $i=1 \sim n$, and here are $(m-q)$ species expressed as set:

$$
C=\left\{c_{p}\right\}, p=m \sim q, C \subseteq B
$$

We define $\alpha_{p}$ as Interspecific Interference Ratio (IIR):

$$
\alpha_{p}=\frac{\sum_{r_{c_{j}}>r_{c_{p}}} r_{c_{j}}}{\sum_{k=m}^{q} r_{c_{p}}}, c_{j} \in C
$$

where $\sum_{r_{c_{j}}>r_{c_{p}}} r_{c_{j}}$ is the sum of GRD of the single specie whose correlation degree is larger. $\sum_{k=m}^{q} r_{c_{p}}$ is the sum of GRD of all the species in the environment.

We add factor IIR to reflect interactions between fungi species, then the decomposition rate is expressed as follows:

$$
R_{\text {interaction }}=\left(1-\alpha_{p}\right) \cdot R_{d e}
$$

\subsection{Characterization of the dynamics of interactions including short- and long-term trends}

With global change rapidly, it's necessary to build a more flexible model using long-term data to predict the effect of ecological performance traits accurately. From the perspective of ecology, temperature will influence the enzyme activity and has direct impact on the decomposition rate. Meanwhile, temperature can affect the growth rate of fungi and has indirect impacts on the decay rate. Moisture content in the environment can influence the viability of fungi. That's why temperature and moisture content per volume are viewed as main affecting factors in the long-term model ${ }^{[3]}$. Our model to reveal long-term trends is based on the equation implemented by Brad Oberle.

In the case of temperature, a logistic increase of decomposition rate was used as shown in Equation $(13)^{3}$ :

$$
T_{1}=\left\{\begin{aligned}
\frac{1}{1+b \cdot e^{-c \cdot T}}, & T<T_{O} \\
e^{-\left(\frac{T-a}{d}\right)^{2}}, & T_{O}<T<40^{\circ} \mathrm{C}
\end{aligned}\right.
$$

$T_{1}$ is the scalar factor limiting decay rate as a function of temperature. $T_{O}$ is the temperature when the decomposition rate reaches the highest point and it is also called optimal temperature. $b, c, d$ are empirical coefficients determined by the boundary conditions. According to our dataset, we have:

$$
T_{1}=\left\{\begin{aligned}
\frac{1}{1+100 \cdot e^{-\frac{14}{T_{O}} \cdot T},} & T<T_{O} \\
e^{-\left(\frac{-T-T_{O}}{10}\right)^{2}}, & T_{O}<T<40^{\circ} \mathrm{C}
\end{aligned}\right.
$$

In the case of moisture content, a gaussian increase and a linear decrease of decomposition rate were used ${ }^{3}$. After calibration, we get:

$$
T_{2}= \begin{cases}(2.4-0.25 O M) \mathrm{e}^{-\left[\frac{y-(O M+0.2)}{0.22}\right]^{2}} & 0 \leq y<O M \\ -\frac{1}{1-O M} y+\frac{1}{1-O M} & O M<y \leq 1\end{cases}
$$

$T_{2}$ is the scalar factor limiting decay rate as a function of moisture contents. $O M$ is the optimal moisture content. From our dataset, we choose the data of 14 isolates which live in the same condition and employ average optimal temperature and average optimal moisture contents to determine scalar factor, so $T_{O}=28.04^{\circ} \mathrm{C}$ and $O M=$ $52.14 \%$. Substitute those two values into Equation (14) $\& 15$ respectively, we yield the Figure 2 as follows:

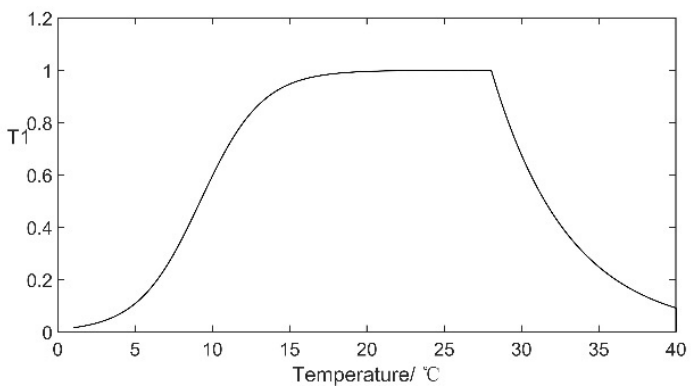

(A)

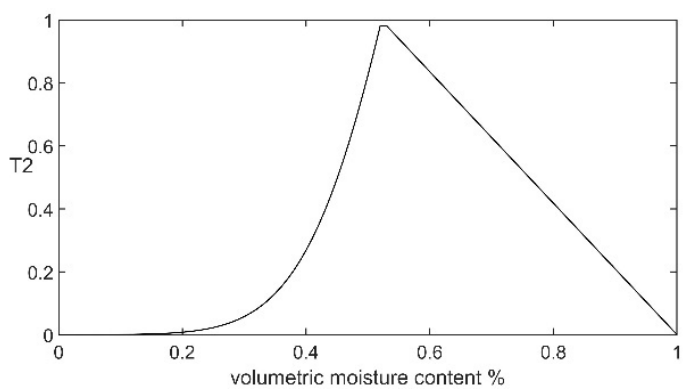

(B)

Fig. 2. Shape of scalar factors limiting decomposition rates as function of (A) temperature and (B) moisture content in longterm models

After analyzing the location distribution of these 34 fungi isolates, we found 14 isolates are clustered around the Great Lakes. It means that environment has same impacts on these 14 isolates, so we draw the decomposition rate curve with respect to time.

From Equation (8), we get the decomposition rate of single fungi specie. However, when consider ing interspecific effect, there will be logistic increment of the decay rate as shown in Equation (14) and (15), so we use scale factor $T_{1}, T_{2}$ to modify the Equation (8) and compute: 


$$
R_{d e}^{\prime}=e^{l n\left(R_{d e}\right)+T_{1}+T_{2}}
$$

When the mean environment temperature $T=10^{\circ} \mathrm{C}$ and mean moisture contents $y=0.65$, we draw the decomposition rate curve without interactions as shown in Figure 3.

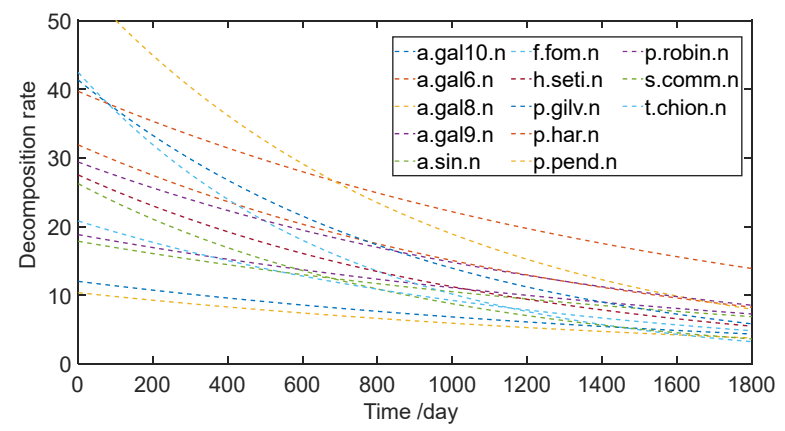

Fig. 3. Decomposition rate of 14 isolates without interactions in the long term. Average environment temperature $T=10^{\circ} \mathrm{C}$ and mean moisture contents $y=0.65$

Then, by substituting Equation (16) into (17) to compute, we get the dynamic model of long-term interactions. When the mean environment temperature $T=10^{\circ} \mathrm{C}$ and mean moisture contents $y=0.65$, we draw the decomposition rate curve with interactions as shown in Figure 4.

$$
R_{\text {interaction }}^{\prime}=\left(1-\alpha_{p}\right) \cdot R_{d e}^{\prime}
$$

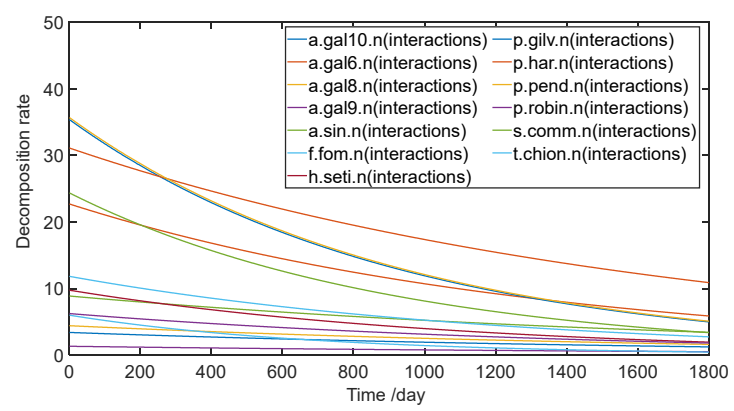

Fig. 4. Decomposition rate of 14 isolates with long-term interactions. Average environment temperature $T=10^{\circ} \mathrm{C}$ and mean moisture contents $y=0.65$

Compare Figure 3 and 4, we will find that in the longterm trends, interactions between different fungi species will suppress the activity of fungi. Some biologists call this phenomenon interspecific competition. ${ }^{[4]}$ In Figure 3, decomposition curves of 14 isolates are almost evenly distributed; however, in Figure 4, the curves of most species are densely distributed at a relative low level. In the competition, a few species are dominant, and decomposition remains in a relative high level, since they are better adapted to the environment when resources are limited, which also follows the general evolution theory of survival for the fittest.

\section{Prediction in varied climate and role of biodiversity}

In this part, we analyze the diversity of climate and explore the role of biodiversity in various environment. We found that the more complex the ecosystem is, and the more biological species are, the higher the stability of the ecosystem is.

\subsection{Prediction of Species' advantages and disadvantages}

According to the dataset, 20 species (34 isolates) are collected from Alaska to Puerto Rico, and the climate of those places varies greatly. We mark those sites on the map according to the exact longitude and latitude values as shown in Figure 5.

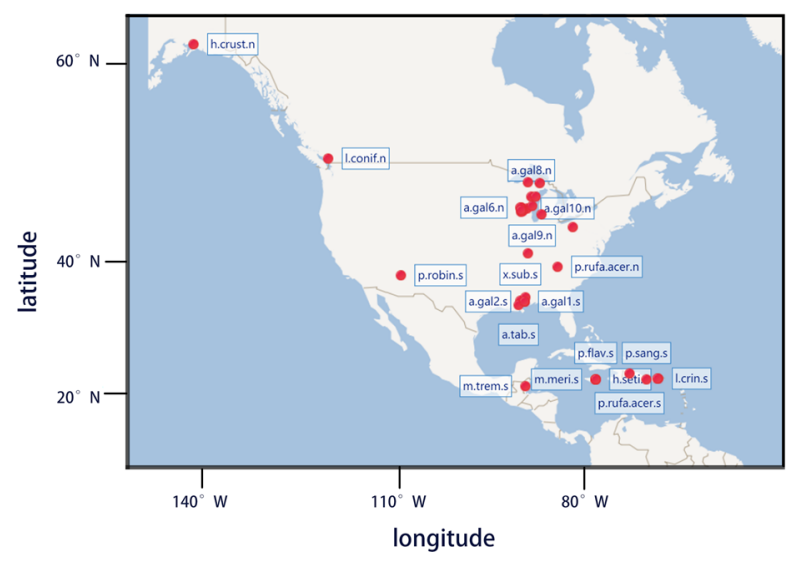

Fig. 5. The spatial distribution of 34 isolates. Climate conditions where the fungi from influence the decomposition rate greatly.

We classify the 34 isolates into 5 types according to their location. Use Equations (12) and (17) to draw the curve of decomposition rate with respect of different climates, as shown in Figure 6:

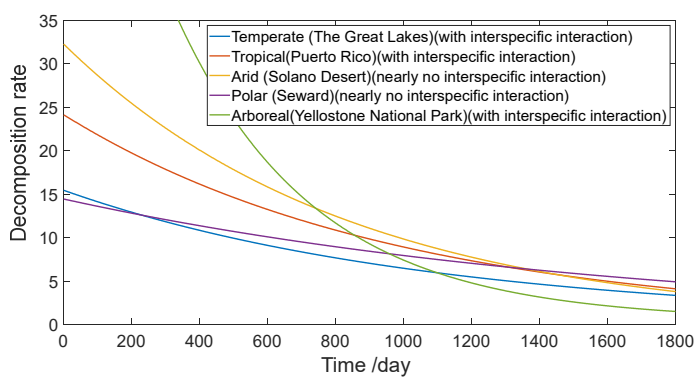

Fig. 6. The curve of decomposition rate of fungi in different climate types. Fungi from Yellowstone National Park (optimal temperature is $29^{\circ} \mathrm{C}$ and optimal moisture content 0.397 ) have the highest decomposition rate because the optimal moisture content is close to the ambient moisture content.

So far, we have built the model of decomposition with respect to time in both short- and long-term trends. We also explore the interactions between fungi species using Grey System Theory, and define $\alpha_{p}$ as Interspecific Interference Ratio (IIR), to evaluate the relative advantages and disadvantages, we define $1-\alpha_{p}$ as the Relative Interspecies Competitiveness (RIC). According to Equation (11), we can compute the value of RIC shown in Figure 7. 


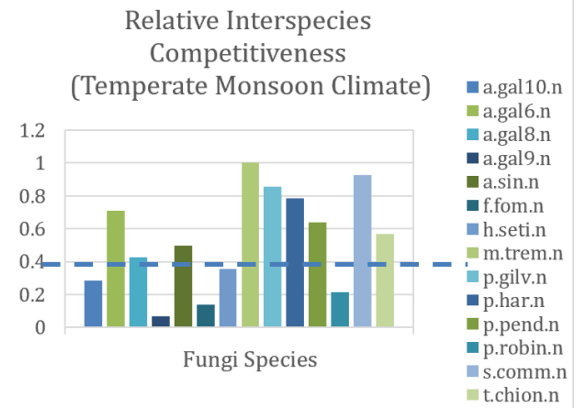

(A)

Relative Interspecies

Competitiveness

(Tropical Rainforest Climate)

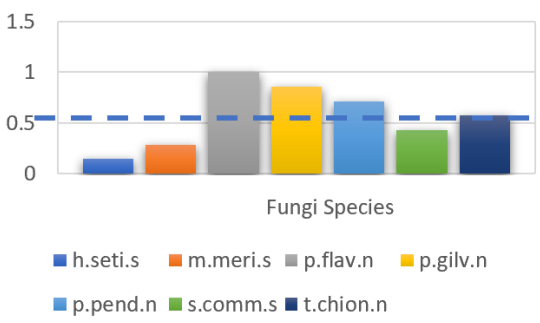

(B)

Fig. 7. The evaluation competitiveness for species in (A)Temperate Monsoon Climate and (B) Tropical Rainforest Climate

From Figure 7, it's clear that in Temperate Monsoon Climate, m.trem.n is the most competitive specie, while in tropical rainforest climate, p.flav.n is the most competitive one.

\subsection{Prediction of role of biodiversity under different environmental variability}

Different fungal colonies have different decomposition rates for different materials, and the diversity of fungal communities can establish a small ecological circle, which influences each other to promote the accelerated decomposition of litters. For example: the proliferating fungi produced by compostable litters accelerates the decomposition of organic matters in litters, releases energy to kill germs and insect eggs, and produces farmyard manure and other resources for fuel or power generation. ${ }^{[4]}$

In our model, we see that the decomposition rate of a population of fungi is faster than that of any single fungus. (see Fig. 8.) The diversity of fungal communities greatly improves the efficiency of decomposition.

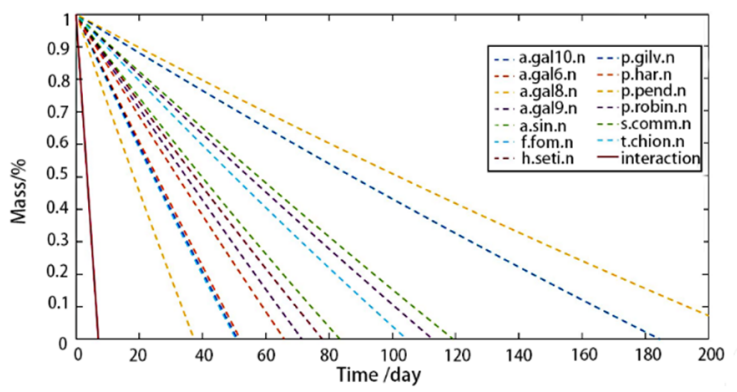

Fig. 8. The effect of biodiversity on the ecosystem
The resistance stability of the ecosystem is determined by the automatic adjustment ability of the ecosystem, and ecosystems with large automatic adjustment ability have high resistance stability. The auto-regulation ability is related to the complexity of the ecosystem. The more complex the ecosystem is and the more biological species are, the greater the auto-regulation ability is, the higher the stability of the ecosystem's resistance is, and the less susceptible the ecosystem is to external interference.

In the presence of different degrees of variability in the local environment, the diversity of fungi in areas such as ice fields and deserts are low, their anti-interference ability is relatively poor, and their resilience is also worse, but the diversity of fungi in areas such as freshwater lakes and tropical rain forests is relatively high with strong antiinterference ability and stronger resilience.

\section{Sensitivity and robustness analysis}

\subsection{Sensitivity analysis}

As we mentioned before, environmental conditions have huge impact on the decomposition rate of fungi. ${ }^{[3]}$ The main affecting factors of our long-term model are temperature and moisture contents, and sensitivity analysis is needed to test the reliability of this model. We choose Relative Error (RE) as the indicator of sensitivity:

We choose h.seti.n specie to represent the average level of the whole 14 isolates, and the average annual temperature of the Great lakes is $10^{\circ} \mathrm{C}$ and mean moisture content is 0.65 , so we perturb the temperature and humidity in the extent of $\pm 2 \%$, to observe the change of decomposition rate. The results of sensitivity analysis are shown in Figure 9 and Figure 10 respectively.
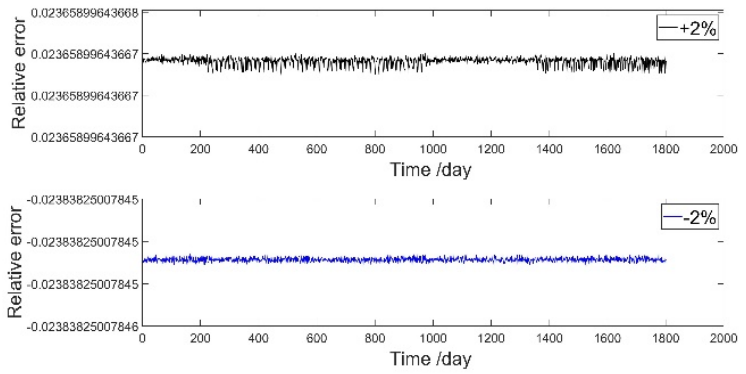

Fig. 9. Sensitivity analysis of rapid fluctuations in temperature
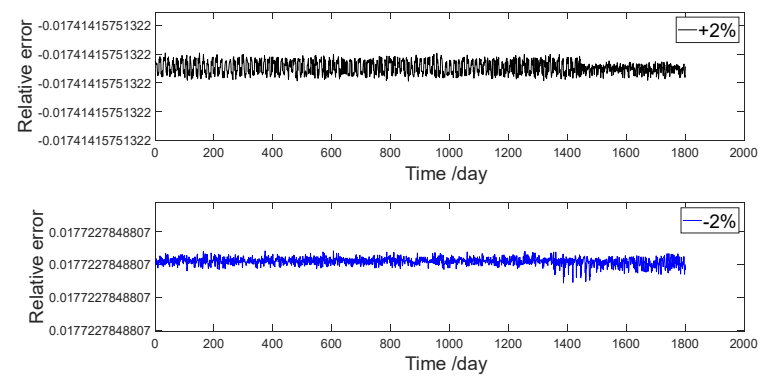

Fig. 10. Sensitivity analysis of rapid fluctuations in moisture

When the fluctuation is $\pm 2 \%$, the variation of decomposition rate is about $\pm 2.36 \%$, which means that 
our model is quite robust and will not change greatly due to the perturbation of the initial value.

\subsection{Robustness analysis}

It's significant to test whether the final decomposition rate can be stable under different fungi species. The decomposition rate of fungi species can be written as $\Phi_{i}(x, y \mid x, y \in \Omega)$. The degree of fit $R M S E_{i j}$ is calculated by:

$$
R M S E_{i j}=\sqrt{\frac{1}{N} \sum_{(x, y \in \Omega)}\left[\Phi_{i}(x, y)-\Phi_{j}(x, y)\right]^{2}}
$$

Then, we draw the Q-Q plot of RMSE versus Standard Normal:

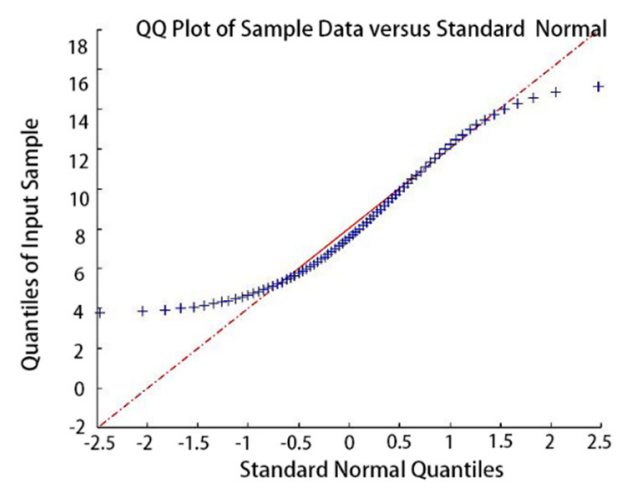

Fig. 11. Q-Q plot of RMSE versus Standard Normal

From Q-Q plot, we see that the scattered points of the plot are distributed roughly on the straight line, which shows that our fit situation is stable. When o combination of species changed, similar decomposition results can be obtained, which reveal the robustness of our model.

\section{Conclusions and improvement}

We focus on the wood decomposer fungi using negative exponent model to express the mass loss as a function of time, then derivate this formula to compute the decomposition rate of multiple species.

For short-term trend, we define Interspecies Interference Ratio (IIR) and rank relative competitiveness based on Grey System Theory with four evaluation indices - hyphal extension rate, moisture niche width, the hyphal density and thermal niche width. For long-term trend, temperature and moisture content per volume are main affecting factors. Several logistic increase due to fluctuations in the environment are included. Then, we analyze and predict the advantages and disadvantages of fungal communities in different areas such as tropical rain forests, forests, lakes, and deserts by Interspecific Interference Ratio. Finally, we draw the conclusion that the diversity of fungal communities will promote the efficiency of the breakdown of ground litter, as shown in Figure 8.

For further study, we may perform holistic analysis of indirect and direct environmental effect on the decomposition rate. We may also do the analysis of deposition ratio of fungi can be more accurate if we have more complete experiment data.

\section{Acknowledgments}

Thanks to SiYu Liu, RuiXi Wang, and LuYao Dong. They contribute equally to this work.

\section{References}

1. Nicky Lustenhouwer, Daniel S. Maynard, Mark A. Bradford, Daniel L. Lindner, Brad Oberle, Amy E. Zanne, and Thomas W. Crowther, "A trait-based understanding of wood decomposition by fungi," Proceedings of the National Academy of Sciences of the United States, May 13, 2020.

2. Brad Oberle, Marissa R. Lee, Jonathan A. Myers, Oyomoare L. Osazuwa-Peters, Marko J. Spasojevic, Maranda L. Walton, Darcy F. Young, Amy E. Zanne, "Accurate forest projections require long-term wood decay experiments because plant trait effects change through time," Glob Change Biol. 2020;26:864-875.

3. Guido Incerti, Giuliano Bonanomi, Francesco Giannino, Flora Angela Rutigliano, Daniela Piermatteo, Simona Castaldi, Anna De Marco, Angelo Fierro, Antonietta Fioretto, Oriana Maggi g, Stefania Papa, Anna Maria Persiani g, Enrico Feoli, Amalia Virzo De Santof, Stefano Mazzoleni, “Litter decomposition in Mediterranean ecosystems: Modelling the controlling role of climatic conditions and litter quality." Applied Soil Ecology. 49 (2011) 148-157

4. D. S. Maynard et al., Consistent trade-offs in fungal trait expression across broad spatial scales. Nat. Microbiol. 4, 846-853 (2019)

5. "Climate Change; Investigators at University of New South Wales Report New Data on Climate Change (Scientists' warning to humanity: microorganisms and climate change)." Global Warming Focus. (2019). doi:10.1038/s41579-019-0222-5.

6. Amy E. Zanne, et al. "A deteriorating state of affairs: How endogenous and exogenous factors determine plant decay rates." Journal of Ecology 103.6(2015).

7. Kutalik Zoltán, et al. "Connection between stochastic and deterministic modelling of microbial growth." Journal of theoretical biology 232.2(2005).

8. Bradford Mark A, et al. "A test of the hierarchical model of litter decomposition.". Nature ecology \& evolution 1.12(2017).

9. Qichao $\mathrm{Tu}$, et al. "Biogeographic patterns of microbial co-occurrence ecological networks in six American forests." Soil Biology and Biochemistry 148. (2020).

10. Ling-Ling Shi, et al. "Variation in forest soil fungal diversity along a latitudinal gradient." Fungal Diversity 64.1(2014). 